\title{
Coulomb-Type Interaction under Lorentz Symmetry Breaking Effects
}

\author{
R. L. L. Vitória, ${ }^{1}$ H. Belich, ${ }^{2}$ and K. Bakke ${ }^{1}$ \\ ${ }^{1}$ Departamento de Física, Universidade Federal da Paraíba, Caixa Postal 5008, 58051-900 João Pessoa, PB, Brazil \\ ${ }^{2}$ Departamento de Física e Química, Universidade Federal do Espírito Santo, Av. Fernando Ferrari, 514, Goiabeiras, \\ 29060-900 Vitória, ES, Brazil
}

Correspondence should be addressed to K. Bakke; kbakke@fisica.ufpb.br

Received 22 May 2017; Revised 19 July 2017; Accepted 1 August 2017; Published 6 September 2017

Academic Editor: Smarajit Triambak

Copyright (C) 2017 R. L. L. Vitória et al. This is an open access article distributed under the Creative Commons Attribution License, which permits unrestricted use, distribution, and reproduction in any medium, provided the original work is properly cited. The publication of this article was funded by $\mathrm{SCOAP}^{3}$.

Based on models of confinement of quarks, we analyse a relativistic scalar particle subject to a scalar potential proportional to the inverse of the radial distance and under the effects of the violation of the Lorentz symmetry. We show that the effects of the Lorentz symmetry breaking can induce a harmonic-type potential. Then, we solve the Klein-Gordon equation analytically and discuss the influence of the background of the violation of the Lorentz symmetry on the relativistic energy levels.

\section{Introduction}

The search for physics beyond the Standard Model (SM) has been increased with the need for understanding new phenomena, such as the unbalance between matter-antimatter and the dark matter. With respect to the dark matter, it is expected that the dark sector can interact with the visible sector, and thus, it can induce the detection of a weak fifth force in such a way that it can be investigated in decay of an excited state of ${ }^{8} \mathrm{Be}[1]$.

From the studies of quantum chromodynamics (QCD), the size of the proton radius can be estimated through the quark interaction mediated by virtual gluons. Further, experiments with electrons have shown that the value of the size of the proton radius is in agreement with that yielded by the existing theories. However, recent researchers have considered a muon in orbit around a proton and shown that the radius of the proton is different [2]. It is worth pointing out that these facts occurred just when SM goes through its final test: the detection of the Higgs boson [3] at the LHC. Hence, it is necessary to clarify a fundamental question: the Higgs mass. Therefore, these phenomena need a theory that goes beyond SM. In the last decades, the search for a more fundamental theory has given rise to proposal that became known as the Standard Model Extension (SME) [4-6]. An interesting point of SME is that there exist terms that violate the Lorentz symmetry by imposing at least one privileged direction in the spacetime. In recent decades, studies of the violation of the Lorentz symmetry have been made in several branches of physics [7-31].

In this paper, we consider a relativistic scalar particle subject to a scalar potential proportional to the inverse of the radial distance. We also consider this particle to be under the effects of the violation of the Lorentz symmetry. The violation of the Lorentz symmetry is established by a tensor field. Then, we chose a particular background of the violation of the Lorentz symmetry that yields a harmonic-type potential. Thereby, we show that the Klein-Gordon equation can be solved analytically and then discuss the influence of the background of the violation of the Lorentz symmetry on the relativistic energy levels.

The structure of this paper is as follows: in Section 2, we introduce a scalar potential by modifying the mass term of the relativistic equation. We also introduce the background of the Lorentz symmetry violation defined by a tensor field that governs the Lorentz symmetry violation out of SME. Thus, we consider a background of the violation of the Lorentz symmetry yielded by a radial electric field and a nonnull 
component of the Lorentz symmetry breaking tensor and solve the Klein-Gordon equation analytically; in Section 3, we present our conclusions.

\section{Relativistic Effects}

In this section, we consider a relativistic scalar particle subject to a scalar potential proportional to the inverse of the radial distance, and thus, we investigate the effects of a harmonic-type potential produced by an anisotropic environment generated by a violating term of the Lorentz symmetry on this system. We deal with a model that goes for energy scales beyond the Standard Model. In recent years, two of us $[32,33]$ have studied the relativistic quantum dynamics of a scalar particle in a background that breaks the Lorentz symmetry based on the model investigated in $[5,6,18,34,35]$, where a nonminimal coupling is introduced into the Klein-Gordon equation given by $\widehat{p}^{\mu} \widehat{p}_{\mu} \rightarrow \widehat{p}^{\mu} \widehat{p}_{\mu}+$ $(g / 4)\left(K_{F}\right)_{\mu \nu \alpha \beta} F^{\mu \nu}(x) F^{\alpha \beta}(x)$, where $g$ is a constant, $F^{\mu \nu}(x)$ is the electromagnetic tensor, and $\left(K_{F}\right)_{\mu \nu \alpha \beta}$ is the tensor that governs the Lorentz symmetry violation out of SME [5, 6, $29,34]$. From the properties of the tensor $\left(K_{F}\right)_{\mu \nu \alpha \beta}[18]$, it is well-known that it can be decomposed into $3 \times 3$ matrices that give its parity-even sector $\left(\left(\kappa_{D E}\right)_{j k}\right.$ and $\left.\left(\kappa_{H B}\right)_{j k}\right)$ and its parity-odd sector $\left(\left(\kappa_{D B}\right)_{j k}=-\left(\kappa_{H E}\right)_{k j}\right)$ (the matrices of the parity-even sector are defined as $\left(\kappa_{D E}\right)_{j k}=-2\left(K_{F}\right)_{0 j 0 k}$ and $\left(\kappa_{H B}\right)_{j k}=(1 / 2) \epsilon_{j p q} \epsilon_{k l m}\left(K_{F}\right)^{p q l m}$ and are symmetric. On the other hand, the matrices of the parity-odd sector are defined as $\left(\kappa_{D B}\right)_{j k}=-\left(\kappa_{H E}\right)_{k j}=\epsilon_{k p q}\left(K_{F}\right)^{0 j p q}$ and have no symmetry). Thereby, the Klein-Gordon equation can be written in the following form $[18,33,36]$ :

$$
\begin{aligned}
\widehat{p}^{\mu} \widehat{p}_{\mu} \Phi & -\frac{g}{2}\left(\kappa_{D E}\right)_{i j} E^{i} E^{j} \Phi+\frac{g}{2}\left(\kappa_{H B}\right)_{j k} B^{i} B^{j} \Phi \\
-g\left(\kappa_{D B}\right)_{j k} E^{i} B^{j} \Phi & =m^{2} \Phi .
\end{aligned}
$$

On the other hand, as discussed in [37], a scalar potential can be introduced into the Klein-Gordon equation through the modification of the mass term: $m \rightarrow m+S(r, t)$, where $m$ is a constant that corresponds to the mass of the free particle and $S(\vec{r}, t)$ is a scalar potential. It is worth mentioning that, by considering the scalar potential to be $S=S(\vec{r})$, therefore, we build a relativistic position-dependent mass system with the introduction of $S(\vec{r})$ [38-44]. In this work, we consider a scalar potential proportional to the inverse of the radial distance, then, the mass term of the Klein-Gordon equation becomes

$$
m(r)=m+\frac{\chi}{r},
$$

where $\chi$ is a constant that characterizes the scalar potential $S(\vec{r})$. It has been studied in models for confinement of quarks [38], in condensed matter physics [45], and in the cosmic string spacetime [46]. In this work, we work with the Minkowski spacetime in cylindrical coordinates and the units $\hbar=c=1$.

$$
d s^{2}=-d t^{2}+d r^{2}+r^{2} d \varphi^{2}+d z^{2} .
$$

Now, let us consider a background of the Lorentz symmetry violation determined by the presence of the electric field $\vec{E}=(\lambda r / 2) \widehat{r}$, where $\lambda$ is a constant related to a uniform volume distribution of electric charges [33]. In this way, since the component of the Lorentz symmetry breaking tensor $\left(\kappa_{D E}\right)_{11}$ can be considered to be a constant (note that, in this work, the component of the Lorentz symmetry breaking tensor $\left(\kappa_{D E}\right)_{11}$ is considered to be constant in the coordinate system determined by the line element (3), i.e., in cylindrical coordinates. There is nothing that forbids this assumption. However, if one changes the coordinate system, for instance, to Cartesian coordinates, then, this component of the Lorentz symmetry breaking tensor becomes a term that depends on the position. Therefore, by changing to Cartesian coordinates, it represents a soliton-like coefficient that varies with the position and breaks the momentum conservation), the KleinGordon equation (1) becomes

$$
\begin{aligned}
\left(m^{2}+\right. & \left.\frac{2 m \chi}{r}+\frac{\chi^{2}}{r^{2}}\right) \Phi \\
= & -\frac{\partial^{2} \Phi}{\partial t^{2}}+\frac{\partial^{2} \Phi}{\partial r^{2}}+\frac{1}{r} \frac{\partial \Phi}{\partial r}+\frac{1}{r^{2}} \frac{\partial^{2} \Phi}{\partial \varphi^{2}}+\frac{\partial^{2} \Phi}{\partial z^{2}} \\
& -\frac{g\left(\kappa_{D E}\right)_{11} \lambda^{2} r^{2}}{8} \Phi .
\end{aligned}
$$

Observe that the last term of the right-hand side of the Klein-Gordon equation (4) gives rise to a harmonictype potential [33]. Note that this system has the cylindrical symmetry; therefore, the eigenvalues of the $z$-component of the angular momentum operator and the $z$-component of the linear momentum operator are conserved quantities. This allows us to write the solution to (4) in the form $\Phi(t, r, \varphi, z)=e^{-i \mathscr{E} t} e^{i l \varphi} e^{i p_{z} z} f(r)$, where $l=0, \pm 1, \pm 2, \pm 3, \ldots$ are the eigenvalues of the $z$-component of the angular momentum operator and $p_{z}=$ const are the eigenvalues of the $z$-component of the linear momentum operator. In the following, let us define the parameters:

$$
\begin{aligned}
\omega^{2} & =\frac{g\left(\kappa_{D E}\right)_{11} \lambda^{2}}{8} ; \\
\theta & =2 m \chi ; \\
\beta & =\mathscr{E}^{2}-m^{2}-p_{z}^{2} .
\end{aligned}
$$

We proceed with a change of variables given by $\xi=\sqrt{\omega} r$, and thus, we rewrite (4) in the form

$$
f^{\prime \prime}+\frac{1}{\xi} f^{\prime}-\frac{\left(l^{2}+\chi^{2}\right)}{\xi^{2}} f-\xi^{2} f-\frac{\theta}{\sqrt{\omega} \xi} f+\frac{\beta}{\omega} f=0 .
$$

By imposing that when $\xi \rightarrow \infty$ and $\xi \rightarrow 0$, then $f(\xi) \rightarrow$ 0 , therefore the function $f(\xi)$ can be written in terms of an unknown function $h(\xi)$ as follows:

$$
f(\xi)=\xi^{\sqrt{l^{2}+\chi^{2}}} e^{-\xi^{2} / 2} h(\xi) .
$$


Next, by substituting (7) into (6), we find out that the function $h(\xi)$ is a solution to the second order differential equation:

$$
\begin{aligned}
h^{\prime \prime}+ & {\left[\frac{1+2 \sqrt{l^{2}+\chi^{2}}}{\xi}-2 \xi\right] h^{\prime} } \\
& +\left[\frac{\beta}{\omega}-2-2 \sqrt{l^{2}+\chi^{2}}-\frac{\theta}{\sqrt{\omega} \xi}\right]=0 .
\end{aligned}
$$

Equation (8) is called in the literature as the biconfluent Heun equation $[46,47]$ and the function $h(\xi)=H_{\mathrm{B}}\left(2 \sqrt{l^{2}+\chi^{2}}\right.$, $0, \beta / \omega, 2 \theta / \sqrt{\omega} ; \xi)$ is the biconfluent Heun function.

Let us write $h(\xi)=\sum_{k=0}^{\infty} b_{k} \xi^{k}$, which means that $h(\xi)$ is written as a power series expansion around the origin $[36,48]$. Thereby, we substitute $h(\xi)=\sum_{k=0}^{\infty} b_{k} \xi^{k}$ into (8) and obtain the relation

$$
b_{1}=\frac{\theta}{\sqrt{\omega}\left(1+2 \sqrt{l^{2}+\chi^{2}}\right)} b_{0}
$$

and the recurrence relation

$$
b_{k+2}=\frac{\theta b_{k+1}+\sqrt{\omega}\left(2 k+2+2 \sqrt{l^{2}+\chi^{2}}-\beta / \omega\right) b_{k}}{\sqrt{\omega}(k+2)\left(k+2+2 \sqrt{l^{2}+\chi^{2}}\right)} .
$$

We go further in search of bound state solutions; thus, we must impose that the biconfluent Heun series terminates. This occurs, from (10), when

$$
\begin{aligned}
\frac{\beta}{\omega}-2-2 \sqrt{l^{2}+\chi^{2}} & =2 n \\
b_{n+1} & =0
\end{aligned}
$$

where $n=1,2,3, \ldots$, which means that the biconfluent Heun series becomes a polynomial of degree $n$ when the two conditions given in (11) are satisfied. From the condition $\beta / \omega-2-2 \sqrt{l^{2}+\chi^{2}}=2 n$, we obtain

$$
\mathscr{E}_{n, l}= \pm \sqrt{m^{2}+2 \omega\left(n+\sqrt{l^{2}+\chi^{2}}+1\right)+p_{z}^{2}}
$$

which corresponds to the relativistic energy levels of the system. Observe that $n$ is the quantum number related to the radial modes.

Furthermore, in order that the biconfluent Heun series terminates, we also need to analyse the condition $b_{n+1}=0$ given in (11). For this purpose, let us return to the series $h(\xi)=\sum_{k=0}^{\infty} b_{k} \xi^{k}$ and obtain the first three terms of it. We start with $b_{0}=1$ and then, from (9) and (10), we have

$$
\begin{aligned}
b_{1}= & \frac{\theta}{\sqrt{\omega}\left(1+2 \sqrt{l^{2}+\chi^{2}}\right)} \\
b_{2}= & \frac{\theta^{2}}{2 \omega\left(2+2 \sqrt{l^{2}+\chi^{2}}\right)\left(1+2 \sqrt{l^{2}+\chi^{2}}\right)} \\
& -\frac{9}{2\left(2+2 \sqrt{l^{2}+\chi^{2}}\right)}
\end{aligned}
$$

where $\vartheta=\beta / \omega-2-2 \sqrt{l^{2}+\chi^{2}}$. Hence, by dealing with the lowest energy state of the system $(n=1)$, we have that $b_{n+1}=$ $b_{2}=0$. From this condition, we can express the parameter $\chi$ that characterizes the Coulomb-type scalar potential in terms of the parameters of the Lorentz symmetry violation, the mass of particle, and the quantum numbers $\{n, l\}$. This relation is obtained by taking the solutions to the following fourth degree algebraic equation for $\chi$ :

$$
\chi_{1, l}^{4}-\left(\frac{\omega}{m^{2}}+\frac{\omega^{2}}{m^{4}}\right) \chi_{1, l}^{2}-\frac{\omega^{2}}{m^{4}}\left(l^{2}-\frac{1}{4}\right)=0,
$$

where we have labelled $\chi_{n, l}$ in order to emphasize that the possible values of this parameter depend on the quantum number $\{n, l\}$. Thereby, the four permitted values of the parameter $\chi_{1, l}$, which is associated with the lowest energy state of the system, are

$$
\begin{aligned}
& \chi_{1, l}^{(1)}=\frac{\sqrt{2}}{2 m^{2}} \sqrt{\omega m^{2}+\omega^{2}+\omega \sqrt{2 \omega m^{2}+\omega^{2}+4 m^{4} l^{2}}} ; \\
& \chi_{1, l}^{(2)}=-\frac{\sqrt{2}}{2 m^{2}} \sqrt{\omega m^{2}+\omega^{2}+\omega \sqrt{2 \omega m^{2}+\omega^{2}+4 m^{4} l^{2}}} ; \\
& \chi_{1, l}^{(3)}=\frac{1}{2 m^{2}} \sqrt{2 \omega m^{2}+2 \omega^{2}-2 \omega \sqrt{2 \omega m^{2}+\omega^{2}+4 m^{4} l^{2}}} ; \\
& \chi_{1, l}^{(4)} \\
& =-\frac{1}{2 m^{2}} \sqrt{2 \omega m^{2}+2 \omega^{2}-2 \omega \sqrt{2 \omega m^{2}+\omega^{2}+4 m^{4} l^{2}}} .
\end{aligned}
$$

In this way, both conditions established in (11) are satisfied, and thus we obtain a polynomial solution to the function $h(\xi)$. With this information, the expression for energy level of the lowest energy state of the system $(n=1)$ is given by

$$
\mathscr{E}_{1, l}= \pm \sqrt{m^{2}+\sqrt{\frac{g\left(\kappa_{D E}\right)_{11} \lambda^{2}}{2}}\left(2+\sqrt{l^{2}+\chi_{1, l}^{2}}\right)+p_{z}^{2}} .
$$

Finally, let us use the label $\chi_{n, l}$ and write (12) in form

$$
\begin{aligned}
& \mathscr{E}_{n, l} \\
& = \pm \sqrt{m^{2}+\sqrt{\frac{g\left(\kappa_{D E}\right)_{11} \lambda^{2}}{2}}\left(n+\sqrt{l^{2}+\chi_{n, l}^{2}}+1\right)+p_{z}^{2}} .
\end{aligned}
$$


From (11) to (17) we can observe that the effects of the Lorentz symmetry violation modify the relativistic energy levels of the Coulomb-type scalar potential $[32,37]$. The influence of the background of the violation of the Lorentz symmetry on the Coulomb-type interaction restricts the parameter $\chi$ to a set of permitted values in order to achieve a polynomial solution to the function $h(\xi)$. As an example, for the lowest energy state of the system, the set of the permitted values of $\chi$ has been given in (15).

\section{Conclusions}

We have investigated relativistic effects on a scalar particle under the influence of a scalar potential proportional to the inverse of the radial distance and the violation of the Lorentz symmetry. We have considered a background of the violation of the Lorentz symmetry that yields a harmonictype potential on the Klein-Gordon equation. Then, in search of polynomial solutions to the function $h(\xi)$ given in (7), we have seen that the effects of the Lorentz symmetry violation modify the relativistic energy levels of the Coulomb-type scalar potential. The influence of the Lorentz symmetry violation background on the Coulomb-type interaction restricts the parameter $\chi$ to a set of permitted values that yield a polynomial solution to $h(\xi)$. As an example, we have obtained the permitted values of $\chi$ for the lowest energy state of the system.

\section{Conflicts of Interest}

The authors declare that they have no conflicts of interest.

\section{Acknowledgments}

The authors would like to thank the Brazilian agencies CNPq and CAPES for financial support.

\section{References}

[1] J. L. Feng, B. Fornal, I. Galon et al., "Protophobic fifthforce interpretation of the observed anomaly in ${ }^{8} \mathrm{Be}$ nuclear transitions," Physical Review Letters, vol. 117, no. 7, Article ID 071803, 2016.

[2] R. Pohl, F. Nez, L. M. P. Fernandes, F. D. Amaro, and F. Birab, "Laser spectroscopy of muonic deuterium," Science, vol. 353, no. 6300, pp. 669-673, 2016.

[3] D. L. Anderson, M. Sher, and I. Turan, "Lorentz and CPT violation in the higgs sector," Physical Review D, vol. 70, no. 1, Article ID 016001, 2004.

[4] V. A. Kostelecký and S. Samuel, "Spontaneous breaking of Lorentz symmetry in string theory," Physical Review D, vol. 39, no. 2, p. 683, 1989.

[5] D. Colladay and V. A. Kostelecký, "CPT violation and the standard model," Physical Review D, vol. 55, no. 11, Article ID 6760, 1997.

[6] D. Colladay and V. A. Kostelecký, "Lorentz-violating extension of the standard model," Physical Review D, vol. 58, no. 11, Article ID 116002, 1998.
[7] H. Belich, T. Costa-Soares, M. M. Ferreira Jr., J. A. HelayëlNeto, and F. M. O. Moucherek, "Lorentz-violating corrections on the hydrogen spectrum induced by a nonminimal coupling," Physical Review D, vol. 74, no. 6, Article ID 065009, 2006.

[8] G. Gazzola, H. G. Fargnoli, A. P. Baêta Scarpelli, M. Sampaio, and M. C. Nemes, "QED with minimal and nonminimal couplings: on the quantum generation of Lorentz-violating terms in the pure photon sector," Journal of Physics G: Nuclear and Particle Physics, vol. 39, no. 3, Article ID 035002, 2012.

[9] H. Belich, L. P. Colatto, T. Costa-Soares, J. A. Helayël-Neto, and M. T. D. Orlando, "Magnetic moment generation from non-minimal couplings in a scenario with Lorentz-symmetry violation," European Physical Journal C, vol. 62, no. 2, pp. 425432, 2009.

[10] R. Casana, M. M. Ferreira Jr., V. E. Mouchrek-Santos, and E. O. Silva, "Generation of geometrical phases and persistent spin currents in 1-dimensional rings by Lorentz-violating terms," Physics Letters B, vol. 746, pp. 171-177, 2015.

[11] R. Casana, C. F. Farias, and M. M. Ferreira Jr., "Topological selfdual configurations in a Lorentz-violating gauged $O(3)$ sigma model," Physical Review D, vol. 92, Article ID 125024, 2015.

[12] R. Casana, M. M. Ferreira Jr., and F. E. P. dos Santos, "GuptaBleuler quantization of the anisotropic parity-even and CPTeven electrodynamics of a standard model extension," Physical Review D, vol. 90, Article ID 105025, 2014.

[13] R. Casana, M. M. Ferreira Jr., E. da Hora, and A. B. F. Neves, "Maxwell-Chern-Simons vortices in a CPT-odd Lorentzviolating Higgs electrodynamics," The European Physical Journal C, vol. 74, p. 3064, 2014.

[14] R. Casana, M. M. Ferreira Jr., R. V. Maluf, and F. E. P. dos Santos, "Radiative generation of the CPT-even gauge term of the SME from a dimension-five nonminimal coupling term," Physics Letters B, vol. 726, no. 4-5, pp. 815-819, 2013.

[15] H. Belich, T. Costa-Soares, M. M. Ferreira Jr., and J. A. HelayëlNeto, "Non-minimal coupling to a Lorentz-violating background and topological implications," The European Physical Journal C, vol. 41, no. 3, pp. 421-426, 2005.

[16] M. A. Ajaib, "Understanding lorentz violation with rashba interaction," International Journal of Modern Physics A, vol. 27, Article ID 1250139, 2012.

[17] A. G. Grushin and D. Phys. Rev, Phys. Rev. D, vol. 86, Article ID 045001, 2012.

[18] H. Belich, F. J. L. Leal, H. L. C. Louzada, and M. T. D. Orlando, "Investigation of the $K_{F}$-type Lorentz-symmetry breaking gauge models with vortexlike configurations," Physical Review D, vol. 86, no. 12, Article ID 125037, 2012.

[19] R. Casana, M. M. Ferreira Jr., E. Passos, F. E. P. dos Santos, and E. O. Silva, "New CPT-even and Lorentz-violating nonminimal coupling in the Dirac equation," Physical Review D, vol. 87, no. 4, Article ID 047701, 2013.

[20] H. Belich, E. O. Silva, M. M. Ferreira Jr., and M. T. D. Orlando, "Aharonov-Bohm-Casher problem with a nonminimal Lorentz-violating coupling," Physical Review D, vol. 83, no. 12, Article ID 125025, 2011.

[21] L. R. Ribeiro, E. Passos, C. Furtado, and J. R. Nascimento, "Geometric phases modified by a Lorentz-symmetry violation background," International Journal of Modern Physics A, vol. 30, no. 14, Article ID 1550072, 2015.

[22] K. Bakke and H. Belich, "Quantum holonomies based on the Lorentz-violating tensor background," Journal of Physics G: Nuclear and Particle Physics, vol. 40, no. 6, Article ID 065002, 2013. 
[23] K. Bakke and H. Belich, "A Landau-type quantization from a Lorentz symmetry violation background with crossed electric and magnetic fields," Journal of Physics G: Nuclear and Particle Physics, vol. 42, no. 9, Article ID 095001, 2015.

[24] K. Bakke and H. Belich, Spontaneous Lorentz symmetry violation and low energy scenarios, LAMBERT Academic Publishing, Saarbrücken, Germany, 2015.

[25] L. R. Ribeiro, C. Furtado, and E. Passos, "An analogy of the quantum hall conductivity in a Lorentz-symmetry violation setup," Journal of Physics G: Nuclear and Particle Physics, vol. 39, no. 10, Article ID 105004, 2012.

[26] Q. G. Bailey and V. A. Kostelecký, "Signals for Lorentz violation in post-Newtonian gravity," Physical Review D, vol. 74, no. 4, Article ID 045001, 2006.

[27] V. A. Kostelecký and J. D. Tasson, "Prospects for large relativity violations in matter-gravity couplings," Physical Review Letters, vol. 102, no. 1, Article ID 010402, 2009.

[28] V. A. Kostelecký, "Gravity, Lorentz violation, and the standard model," Physical Review D, vol. 69, Article ID 105009, 2004.

[29] A. P. Baêta Scarpelli, H. Belich, J. L. Boldo, L. P. Colatto, J. A. Helayël-Neto, and A. L. M. A. Nogueira, "Remarks on the causality, unitary and supersymmetric extension of the Lorentz and CPT-violating Maxwell-Chern-Simons model," Nuclear Physics B - Proceedings Supplements, vol. 127, pp. 105-109, 2004.

[30] H. Belich, T. Costa-Soares, M. A. Santos, and M. T. D. Orlando, "Violação da simetria de Lorentz," Revista Brasileira de Ensino de Física, vol. 29, no. 1, pp. 57-64, 2007.

[31] S. M. Carroll, G. B. Field, and R. Jackiw, "Limits on a Lorentzand parity-violating modification of electrodynamics," Physical Review D, vol. 41, no. 4, p. 1231, 1990.

[32] K. Bakke and H. Belich, "On a relativistic scalar particle subject to a Coulomb-type potential given by Lorentz symmetry breaking effects," Annals of Physics, vol. 360, pp. 596-604, 2015.

[33] K. Bakke and H. Belich, "On the harmonic-type and linear-type confinement of a relativistic scalar particle yielded by Lorentz symmetry breaking effects," Annals of Physics, vol. 373, pp. 115122, 2016.

[34] V. A. Kostelecký and M. Mewes, "Cosmological constraints on lorentz violation in electrodynamics," Physical Review Letters, vol. 87, no. 25, Article ID 251304, 2001.

[35] V. A. Kostelecký and M. Mewes, "Signals for Lorentz violation in electrodynamics," Physical Review D, vol. 66, no. 5, Article ID 056005, 2002.

[36] R. L. L. Vitória, H. Belich, and K. Bakke, "A relativistic quantum oscillator subject to a Coulomb-type potential induced by effects of the violation of the Lorentz symmetry," European Physical Journal Plus, vol. 132, no. 1, article no. 25, 2017.

[37] W. Greiner, Relativistic Quantum Mechanics: Wave Equations, Springer, Berlin, Germany, 3rd edition, 2000.

[38] C. L. Critchfield, "Scalar potentials in the Dirac equation," Journal of Mathematical Physics, vol. 17, no. 2, p. 261, 1976.

[39] M. K. Bahar and F. Yasuk, "Exact solutions of the massdependent klein-gordon equation with the vector quarkantiquark interaction and harmonic oscillator potential," Advances in High Energy Physics, vol. 2013, Article ID 814985, p. 6, 2013.

[40] H. Panahi and Z. Bakhshi, "Dirac equation with positiondependent effective mass and solvable potentials in the Schrödinger equation," Journal of Physics A: Mathematical and Theoretical, vol. 44, no. 17, Article ID 175304, 2011.
[41] C.-S. Jia and A. de Souza Dutra, "Position-dependent effective mass Dirac equations with $P T$-symmetric and non- $P T$ symmetric potentials," Journal of Physics A: Mathematical and General, vol. 39, no. 38, Article ID 11877, 2006.

[42] O. Mustafa and S. Habib Mazharimousavi, " $\eta$-Weak-pseudohermiticity generators and radially symmetric hamiltonians," International Journal of Theoretical Physics, vol. 47, no. 7, pp. 2029-2037, 2008, 111.

[43] T.-Q. Dai and Y.-F. Cheng, "Bound state solutions of the Klein-Gordon equation with position-dependent mass for the inversely linear potential," Physica Scripta, vol. 79, no. 1, Article ID 015007, 2009.

[44] R. L. L. Vitória and K. Bakke, “Torsion effects on a relativistic position-dependent mass system," General Relativity and Gravitation, vol. 48, 161 pages, 2016.

[45] A. D. Alhaidari, "Solution of the Dirac equation with positiondependent mass in the Coulomb field," Physics Letters A, vol. 322, no. 1-2, pp. 72-77, 2004.

[46] E. R. Figueiredo Medeiros and E. R. Bezerra de Mello, "Relativistic quantum dynamics of a charged particle in cosmic string spacetime in the presence of magnetic field and scalar potential," The European Physical Journal C, vol. 72, Article ID 2051, 2012.

[47] A. Ronveaux, Heun's Differential Equations, Oxford University Press, Oxford, UK, 1995.

[48] G. B. Arfken and H. J. Weber, Mathematical Methods for Physicists, Elsevier Academic Press, New York, NY, USA, 6th edition, 2005. 

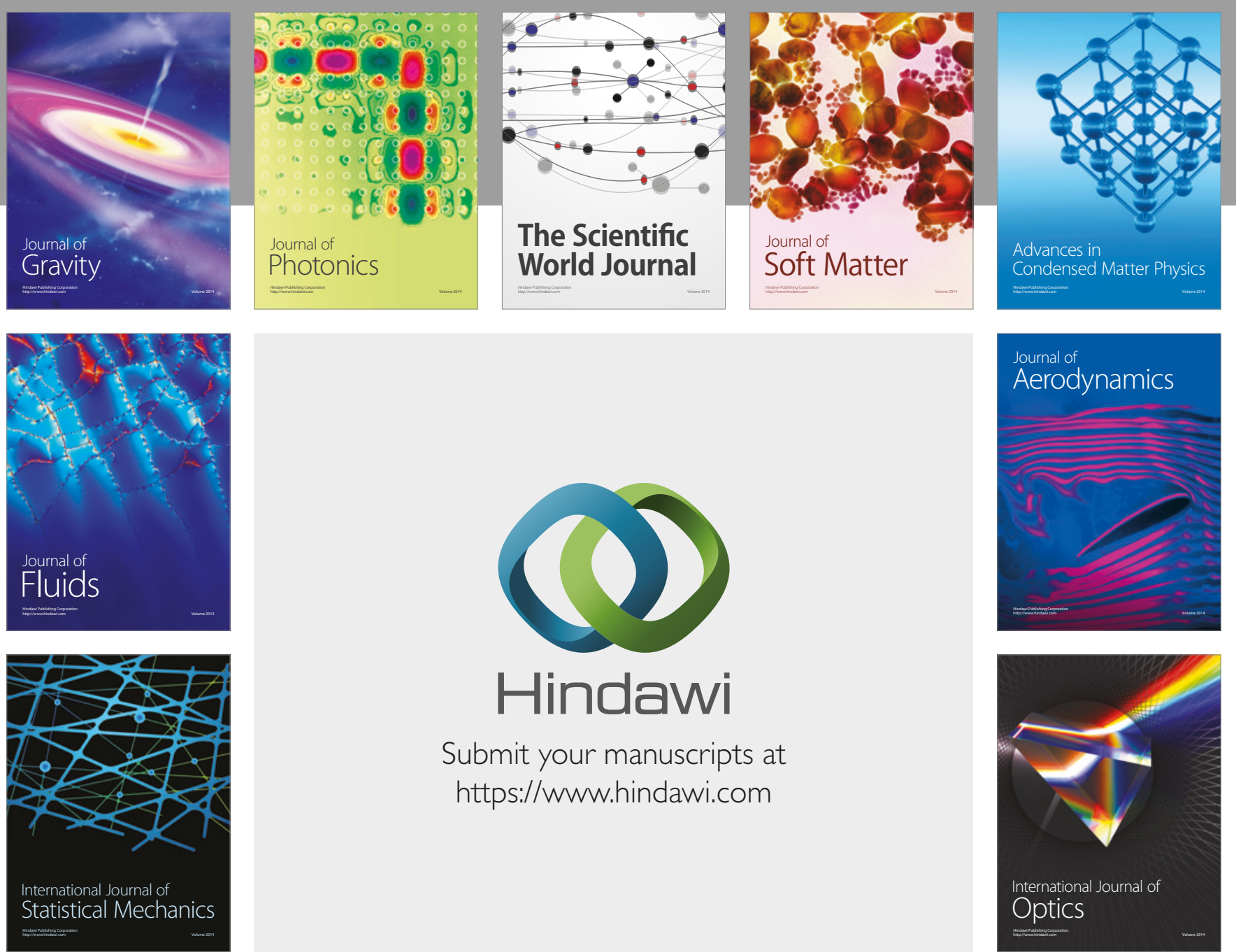

Submit your manuscripts at

https://www.hindawi.com
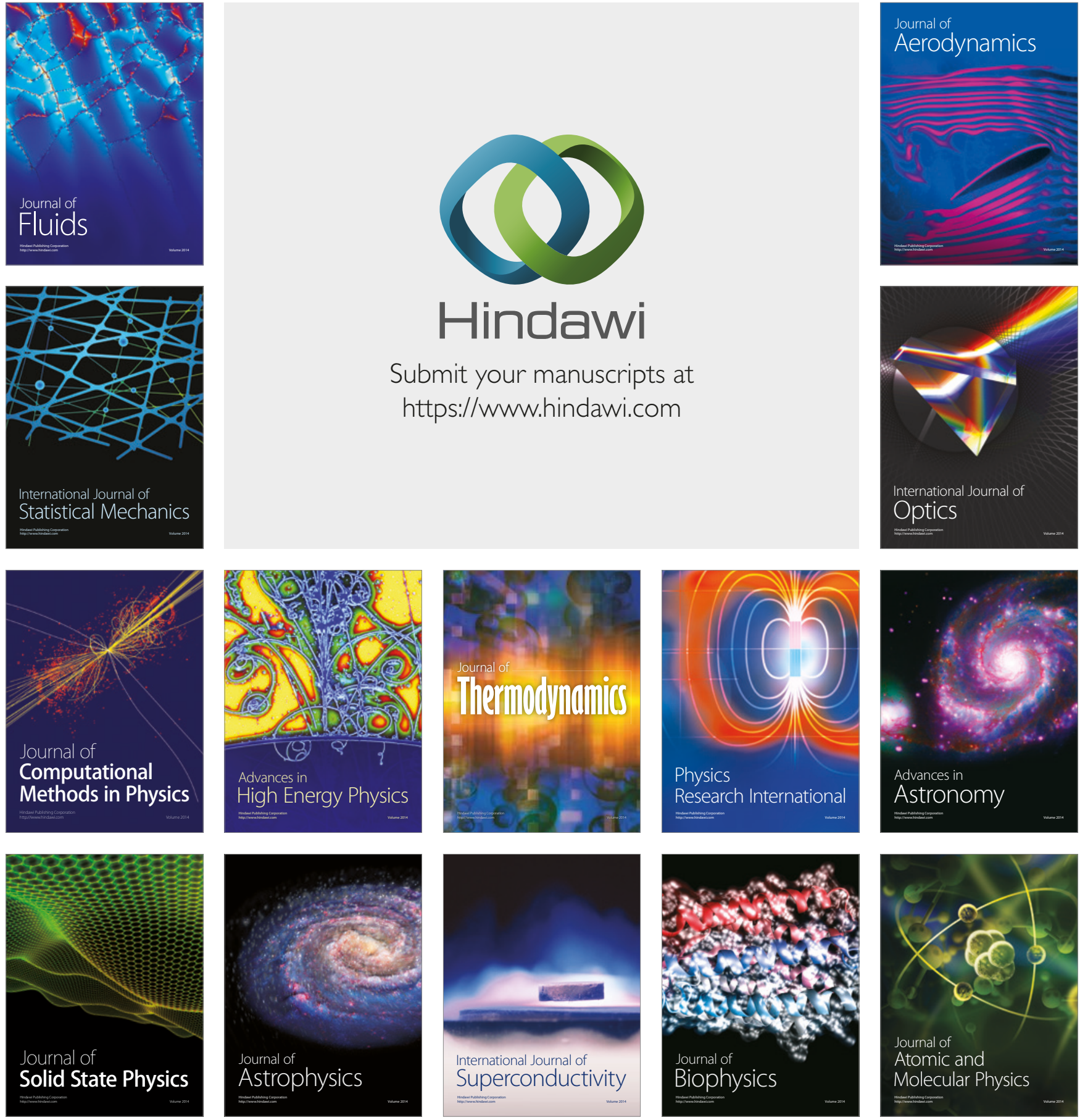